\title{
Heat Kit: Creación de un prototipo para ‘tocar’ el calor.
}

\section{César Martín Gómez ${ }^{a}$, Elia Ibáñez Puy y Amaia Zuazua Ros ${ }^{\mathrm{a}}$}

a Universidad de Navarra, Escuela de Arquitectura, Departamento de Construcción, Instalaciones y Estructuras. Campus Universitario, 31080, Pamplona, España. Tfn. 0034948425600. instetsaun@unav.es

\begin{abstract}
The understanding of heat transfer processes and its affection in buildings is one of the difficulties that the students of architecture have to face when receiving building services courses knowledge, specifically in those related to hygrothermal conditioning.

In a simplified form, it can be said that they must internalise the existence of a heat source, a machine that transfers the heat and an element that absorbs or dissipates the heat in the rooms.

The traditional solution of master classes, visits to real facilities... had a demonstrated effectiveness in the past, however, they fail transferring the implications of thermodynamics in their complexity.

The teaching innovation project entitled "Heatkit - Creation of a prototype to 'touch' the heat", which is the origin of these text, expects that the students of architecture can feel the heat transfer with the construction of a simple and easily replicable thermoelectric prototype.
\end{abstract}

Keywords: Training; arquitecture; engineering; heat transfer; prototype; methodology.

\section{Resumen}

Una de las dificultades de los alumnos de Arquitectura en las asignaturas de instalaciones y concretamente en las que reciben conocimientos de acondicionamiento higrotérmico, es comprender los procesos de transferencia de calor y sus afecciones en el edificio.

De forma simplificada, puede decirse que deben interiorizar que existe una fuente de energía, una máquina que transfiere el calor y un elemento que toma calor, o lo cede, en los locales.

Las soluciones tradicionales de clases magistrales, visitas a instalaciones reales...han demostrado su efectividad en el pasado, sin embargo, no consiguen trasladar en su complejidad las implicaciones de la termodinámica.

Lo que pretende el proyecto de innovación docente titulado "Heatkit Creación de un prototipo para 'tocar' el calor", origen de este texto, es que los alumnos de Arquitectura, puedan sentir la transferencia de calor con la creación de un prototipo termoeléctrico sencillo y fácilmente replicable. 
Palabras clave: Formación; arquitectura; ingeniería; transferencia de calor; prototipo; metodología.

\section{Introducción}

"El bienestar - entendido como respuesta a la temperatura, la humedad, la iluminación e incluso a factores acústicos- ha sido la fuerza motriz en la arquitectura del siglo veinte. Ya estén ocultas, expuestas o de manera integrada, las instalaciones se han convertido en las protagonistas en el ámbito de la construcción” (Granf \& Marino, 2016).

Esta evolución en el ámbito de la Arquitectura es un ejemplo más de que el mundo se encuentra en un cambio constante que también debe hacerse presente en la educación. Así, las escuelas de Arquitectura españolas buscan formar futuros profesionales realmente competentes, capaces de resolver estas nuevas necesidades de la sociedad. Para ello es necesaria una educación integral y significativa, que imparta los conocimientos necesarios buscando alcanzar los niveles superiores del aprendizaje (cfr Monedero Isorna, 2002).

Aumentar la calidad de la enseñanza para alcanzar esta educación integral es una de los principales objetivos de la comunidad educativa. Desde la escuela primaria (Can, 2016), pasando por los institutos (Vega-Moreno, Cufí Solé, José Rueda, \& Llinás, 2016) hasta las universidades (Ben Amara \& Ayari, 2016), la innovación en las aulas se hace cada vez más presente. Nuevas formas de enseñar están siendo desarrolladas (Amran \& Rahman, 2017; De Menezes Maciel, Do Rêgo, \& Carlos, 2017; Deniz \& Simsek, 2015) basadas en estudios que profundizan en la diversidad de los estudiantes (R. M. Felder \& Brent, 2005) y de los tipos de enseñanza y aprendizaje (R. Felder \& Silverman, 1988).

La Escuela de Arquitectura de la Universidad de Navarra, en los últimos años, ha realizado también este esfuerzo por mejorar la calidad de su enseñanza sobre instalaciones. Se han diseñado armarios técnicos dedicados a diferentes conceptos de instalaciones: iluminación de emergencia (Martín-Gómez, Bermejo-Busto, \& Mambrilla-Herrero, 2015), sistemas de climatización, electricidad e incendios (Martín-Gómez, Zapata, Zuazua, Villanueva, \& Olaizola, 2014). Estos armarios funcionan como laboratorios móviles donde el alumno puede observar los sistemas, tocarlos y ponerlos en funcionamiento, acercando los conocimientos teóricos a la realidad.

Un grupo de investigación de la Universidad de Navarra lleva trabajando desde hace años en termoelectricidad, como se detallará más adelante, una tecnología que permite 'visualizar' con gran facilidad los procesos de transferencia de calor. Resultado de estos trabajos son varios prototipos que permiten climatizar un volumen cerrado. Una simplificación de este volumen, para que la transferencia de calor sea fácilmente entendible, montable y transportable por los alumnos, es el objeto del proyecto de innovación docente que se presenta.

(cc) EY-NC-ND 2018, Universitat Politècnica de València

Congreso IN-RED (2018) 


\section{Objetivos}

Lo que pretende el proyecto de innovación docente origen de esta comunicación, es la creación de un prototipo para 'tocar' el calor. Gracias a este prototipo los alumnos de arquitectura, en la asignatura de 'Instalaciones 3', puedan sentir la transferencia de calor, observar la diferencia entre el agua y el aire como medios de disipación, conocer la tecnología Peltier y comprender parámetros eléctricos como la corriente y el voltaje.

Los dos prototipos termoeléctricos utilizados son sencillos y fácilmente replicables, lo que facilita el desarrollo de la sesiones y la interacción de los estudiantes con la termoelectricidad. Además, a través de esta pequeña experiencia se introduce en el aula el mundo de la investigación, despertando en los estudiantes la iniciativa y el espíritu emprendedor que caracteriza a los investigadores y descubriendo una faceta especialmente relevante en el mundo científico.

Se considera que es una técnica educativa innovadora en las escuelas de arquitectura permitiendo trabajar las instalaciones de una manera práctica y que busca fomentar el aprendizaje significativo desde un enfoque transversal que incluye contenidos de diversas materias: ingeniería térmica, transmisión de calor, instalaciones, electricidad, etc.

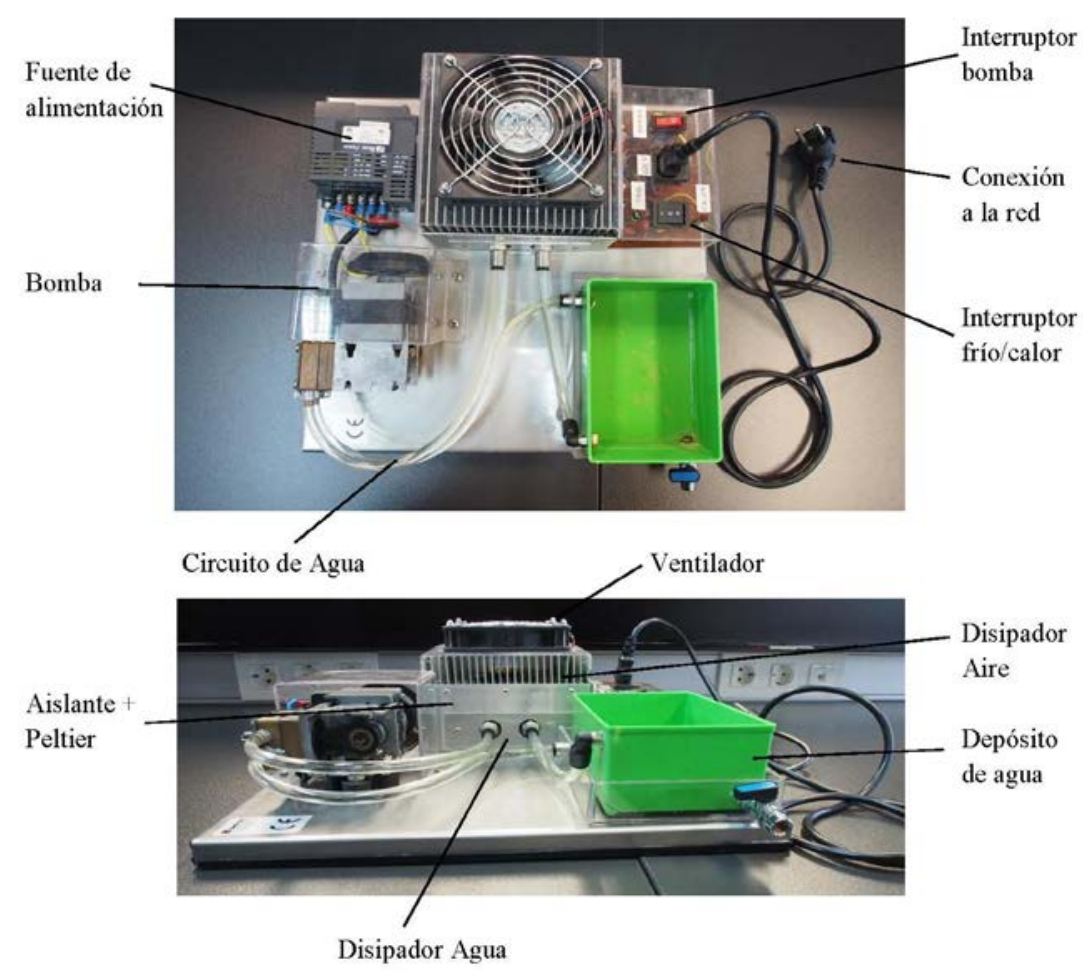

Fig 1. Prototipo de disipación por agua. 


\section{Desarrollo de la innovación}

La idea de esta iniciativa nace del trabajo de un equipo de investigación centrado en el acondicionamiento higrotérmico con termoelectricidad, utilizando células Peltier como medio para la transferencia de calor (Ibáñez-Puy et al, 2014; Ibañez-puy et al, 2017; María Ibañez-Puy et al, 2018; Ibañez-Puy et, 2015; Martín-Gómez et al., 2010). El estudio de esta tecnología, así como su evidente interés docente, han dado como resultado el desarrollo de una sesión práctica que acercan la investigación y la termoelectricidad a los futuros profesionales, al mismo tiempo que les permite interiorizar conceptos básicos para su formación.

La acción educativa consiste en desarrollar una sesión en grupos pequeños con prototipos termoeléctricos, donde se hagan visibles los conceptos de intercambio de calor e ingeniería térmica vistos durante la carrera (Fig. 1).

\subsection{Contenidos}

Como se ha indicado anteriormente las transversalidad es un elemento presente en esta iniciativa, pues aunque la práctica se realice en la asignatura de `Instalaciones 3Acondicionamiento Higrotérmico's se trabajan conceptos de diversos ámbitos.

\subsubsection{Transferencia de calor y acondicionamiento higrotérmico}

Se desarrollan varios conceptos de transferencia de calor:

- $\quad$ Medios de disipación. Se explican dos medios de disipación diferentes, el aire y el agua. Esta característica permite a los estudiantes entender la diferencia entre las dos posibilidades, observar el diferente comportamiento, saber qué implica cada tipología y reflexionar sobre cuál es la mejor solución (Fig. 2).

- $\quad$ Disipador. Se considera importante que los estudiantes toquen y observen in situ diferentes tipologías de disipadores, comprendiendo la justificación de las diferentes morfologías de las aletas, la distancia entre ellas, la rugosidad, etc.

- Termoelectricidad. Es un fenómeno basado en la transferencia de calor. Las células Peltier son capaces de generar una diferencia de temperaturas cuando se les aplica una corriente eléctrica. Es una tecnología en auge que se prevé tenga aplicación en la Arquitectura.

- Concepto de frio y calor. La mayoría de veces los conceptos abstractos de absorber y ceder calor no se comprenden en su totalidad. Con esta experiencia se pretenden materializar estos conceptos ayudando a su comprensión total a través de un aprendizaje significativo.

- $\quad$ Bomba de calor. La célula Peltier puede ser utilizada como bomba de calor. Esta experiencia permite facilitar el tanto el funcionamiento de la bomba como de la termoelectricidad.

(cc) EY-NC-ND 2018, Universitat Politècnica de València

Congreso IN-RED (2018) 


\subsubsection{Electricidad}

A priori, estos conceptos pueden resultar conceptos más propios del mundo de la ingeniería, sin embargo, su comprensión es realmente útil para el funcionamiento de las instalaciones.

- $\quad$ Parámetros eléctricos. El voltaje y la intensidad se conciben como parámetros fundamentales para comprender el funcionamiento de las células Peltier y de cualquier sistema de acondicionamiento higrotérmico.

- $\quad$ Fuente de alimentación. En la sesión los estudiantes pueden tocar y observar de cerca una fuente de alimentación, identificar sus partes y los requisitos para su correcta conexión y funcionamiento.

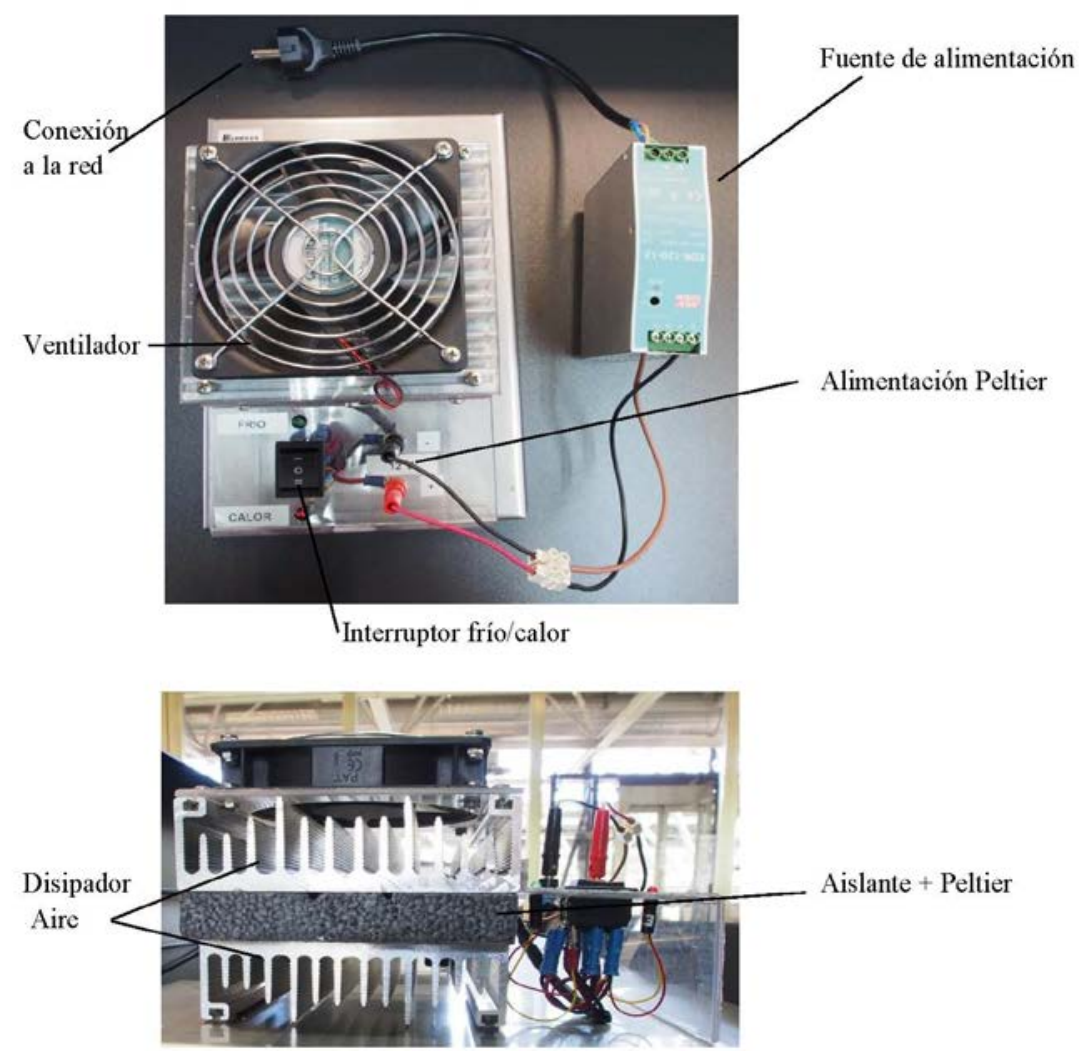

Fig .2 Prototipo de disipación por aire.

\subsection{Metodología}

Para el desarrollo de la sesión se recomienda dividir a los alumnos en grupos reducidos (máximo 10 personas) buscando mejorar la calidad del aprendizaje y el clima de trabajo, favoreciendo la interacción con los prototipos y la resolución de dudas de forma más personalizada. La razón por la que se recomiendan grupos pequeños es facilitar que todos los alumnos puedan participar de manera directa, fomentando el aprendizaje significativo y constructivo (Fig. 3).

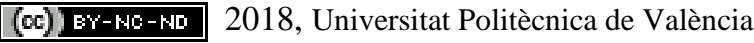


Es fundamental por tanto, desarrollar el papel activo de los alumnos y que sean conscientes de que su aprendizaje depende de su propia implicación en la sesión. El nivel de profundización de los conceptos depende de las preguntas y el interés de los alumnos. De esta forma, se pretende fomentar que los estudiantes sean protagonistas de la sesión.

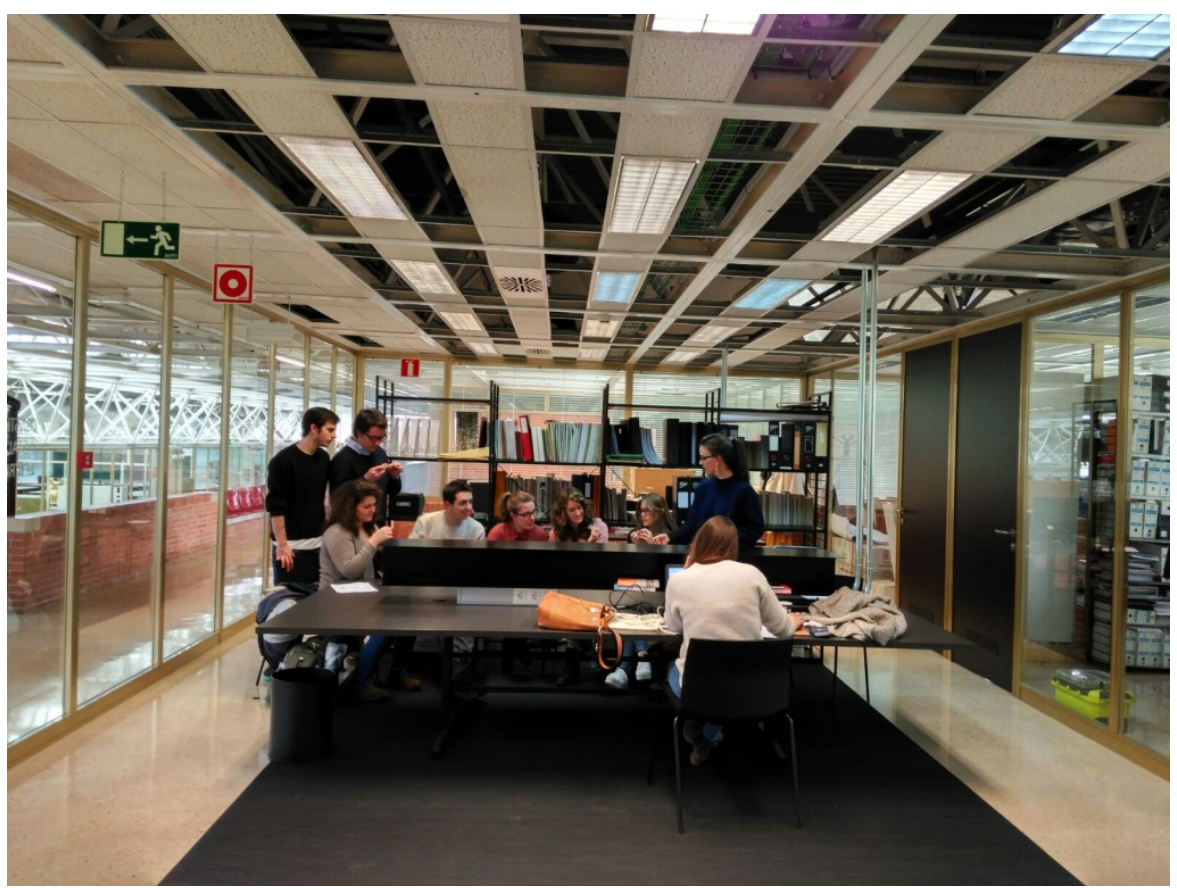

Fig 3. Imagen de una sesión con un grupo de alumnos.

Además, este aprendizaje autorregulado se hace presente durante el desarrollo de toda la práctica donde los alumnos tienen la posibilidad de enchufar, encender y apagar, tocar o cambiar la polaridad de los equipos.

La integración de contenidos de diferentes ámbitos en una única sesión es un ejemplo de la apuesta por la transversalidad docente. Se quiere fomentar que los alumnos no asimilen los conocimientos como estancos, sino que sean capaces de relacionar unos con otros y aplicar la totalidad de su conocimiento ante cualquier situación.

\subsection{Desarrollo de la práctica}

La práctica se realiza con dos prototipos termoeléctricos, uno de aire y uno de agua, que los alumnos deben identificar, poner en marcha y analizar. La sesión se divide en cinco partes:

a) Realización de la encuesta para analizar los conocimientos previos.

b) Parte teórica. Se explican los conceptos básicos que se deben conocer para realizar la práctica.

o Termoelectricidad: fenómeno basado en la transferencia de calor que obtiene una diferencia de temperaturas a partir de la electricidad o viceversa. 
o Célula Peltier: se toca una célula Peltier y el interior de la misma. Se explica su composición y su funcionamiento.

o Medios de disipación de calor: Se explican las diferencias entre el aire y el agua como medio de disipación y se hace reflexionar sobre sus ventajas e inconvenientes.

c) Explicación e identificación de los componentes del sistema.

o Se identifican los dos prototipos termoeléctricos. Se explica cómo están construidos y la localización de las células Peltier.

o Se identifican los disipadores diferenciando los dos medios.

o Se identifica la bomba y las fuentes de alimentación.

d) Parte práctica. Los alumnos ponen en funcionamiento los prototipos y analizan sus comportamientos comentando qué diferencias observan y razonándolas.

o Prototipo de disipación con agua: Se pone en marcha el primero porque su inercia es más lenta y el efecto tarda más en hacerse visible.

o Prototipo de disipación con aire.

o Análisis de los comportamientos, deben reflexionar sobre el comportamiento de los sistemas e identificar en qué posición se encuentra la célula Peltier.

e) Realización de la encuesta final.

Las preguntas o dudas que puedan tener los alumnos se resuelven durante el transcurso de toda la sesión. Se busca fomentar un ambiente de aprendizaje donde el estudiante tenga libertad de realizar las aportaciones que considere importantes durante la práctica.

\section{Resultados}

Para poder valor la eficacia de la sesión los estudiantes realizan una encuesta antes de la sesión y otra después donde se evalúan los conocimientos previos de los estudiantes y su valoración de la prueba. Existe también la posibilidad de añadir propuestas de mejora, de esta forma se han podido identificar aspectos de la práctica que necesitan ser mejorados basándose en la propia experiencia de los estudiantes. Han realizado la encuesta 37 alumnos de Quinto curso de Arquitectura.

La finalidad de las encuestas es alcanzar un primer análisis de la eficacia de la sesión y de poder comprobar su utilidad. Las encuestas presentan pocos ítems (4-5) que se centran en recoger la percepción del estudiante sobre su aprendizaje de los conceptos y valorar si la practica ha servido para mejorarlo (Fig. 4). La mayoría de las preguntas son Sí/No. Existe una sola pregunta de respuesta múltiple para valorar la utilidad de la sesión en su formación y una última pregunta de respuesta libre donde los estudiantes pueden proponer sus mejoras.

Se considera que gracias a estas encuestas se recoge la valoración de los alumnos de manera rápida y eficaz sin invertir mucho tiempo en la recogida de datos. De esta manera, se facilita el análisis de los datos y la incorporación de mejoras casi de manera inmediata.

(cc) EY-Nc-No 2018, Universitat Politècnica de València 
La encuestas se realizan en español y en inglés para los alumnos extranjeros de la escuela así como para que la experiencia sea replicable por otras universidades internacionales.
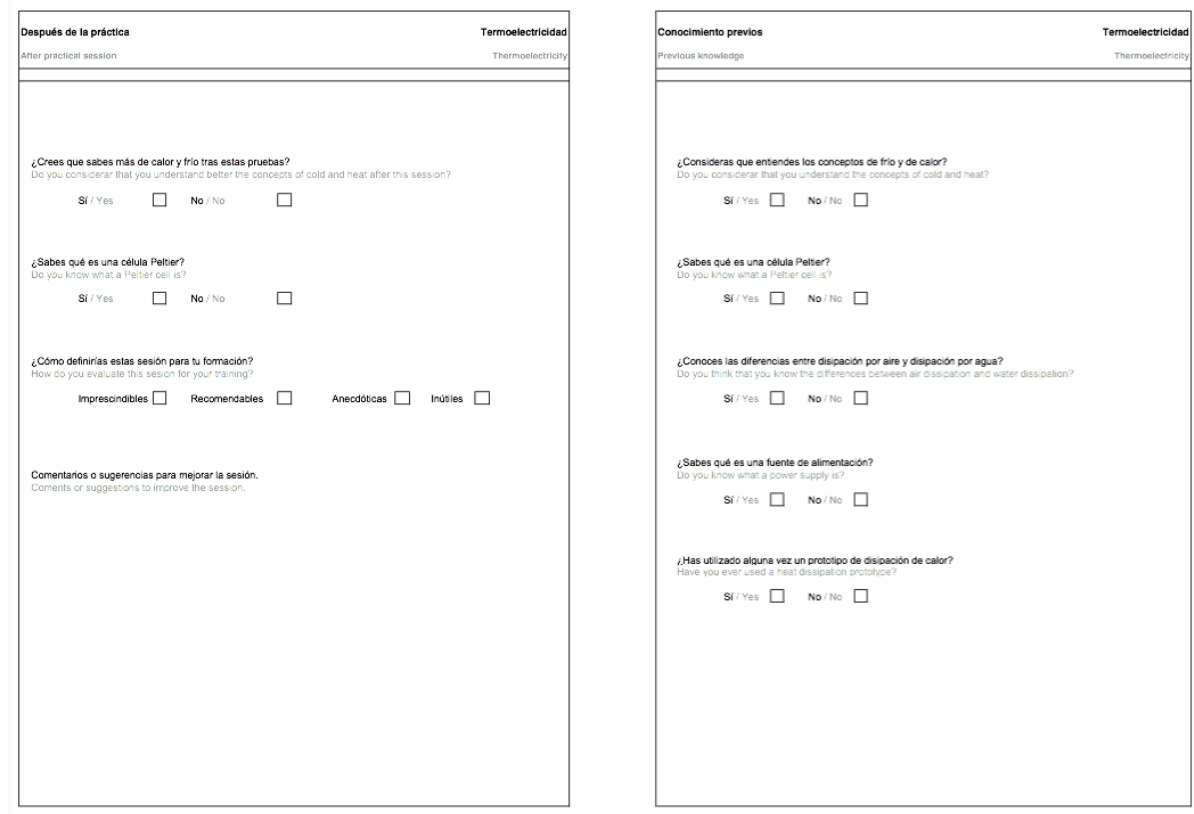

Fig 4. Encuesta de conocimientos previos y de satisfacción realizadas antes y después de la práctica.

Los resultados de la encuesta de conocimientos previos permite analizar qué grado de interiorización de los conceptos se han alcanzado en la asignaturas y además, identificar los puntos fuertes y las áreas de mejora donde se debe incidir en la práctica (Fig. 5).

Se observa que los conceptos de frío y calor y fuentes de alimentación están asentados en el conocimiento de los estudiantes (100\% y 92\%). Sin embargo, un prototipo de disipación y una célula Peltier obtienen los porcentajes más elevados de desconocimiento (84\% y 78\%). La pregunta relacionada con las diferencias entre medios de disipación presenta los resultados más equilibrados de todos los conceptos con un 65\% de alumnos que sí consideran que dominan el concepto.

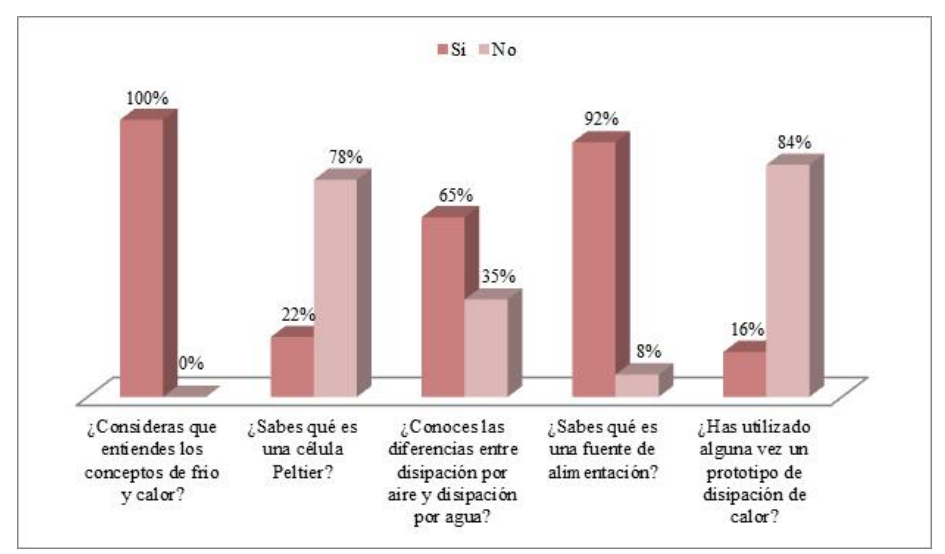

Fig 5. Datos obtenidos en la encuesta de conocimientos previos realizada antes de la sesión.

(c) ) EY-NC-ND 2018, Universitat Politècnica de València

Congreso IN-RED (2018) 
La encuesta realizada tras las pruebas se analiza en dos partes. El primer análisis se centra en la percepción que tiene los alumnos sobre su propio aprendizaje en la sesión. Se puede observar en el diagrama (Fig. 6). Un alto porcentaje de alumnos (96\%) que consideran que ha mejorado su aprendizaje de los conceptos de frío y calor, fundamentales en la asignatura de instalaciones, a pesar de que como se ha comentado anteriormente, todos los alumnos creían que dominaban este concepto antes de la práctica. Del mismo modo, se observa claramente que la sesión permite ampliar los conocimientos sobre células Peltier (96\%).

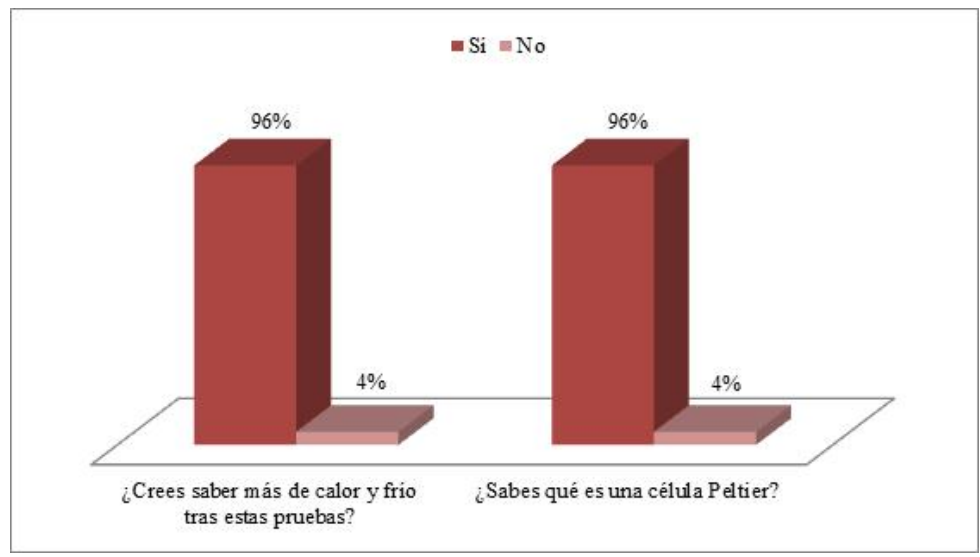

Fig 6. Datos obtenidos tras la realización de la sesión, se valora si los estudiantes han apreciado alguna mejora en el aprendizaje de los conceptos.

La segunda parte del análisis responde a las dos últimas preguntas de la encuesta (Fig. 7). que se centran en la opinión de los estudiantes sobre la sesión y su utilidad para su formación. Un 13\% la considera imprescindible, el mayor porcentaje de alumnos (82\%) considera que la práctica es recomendable y otro $4 \%$ la valora como anecdótica.

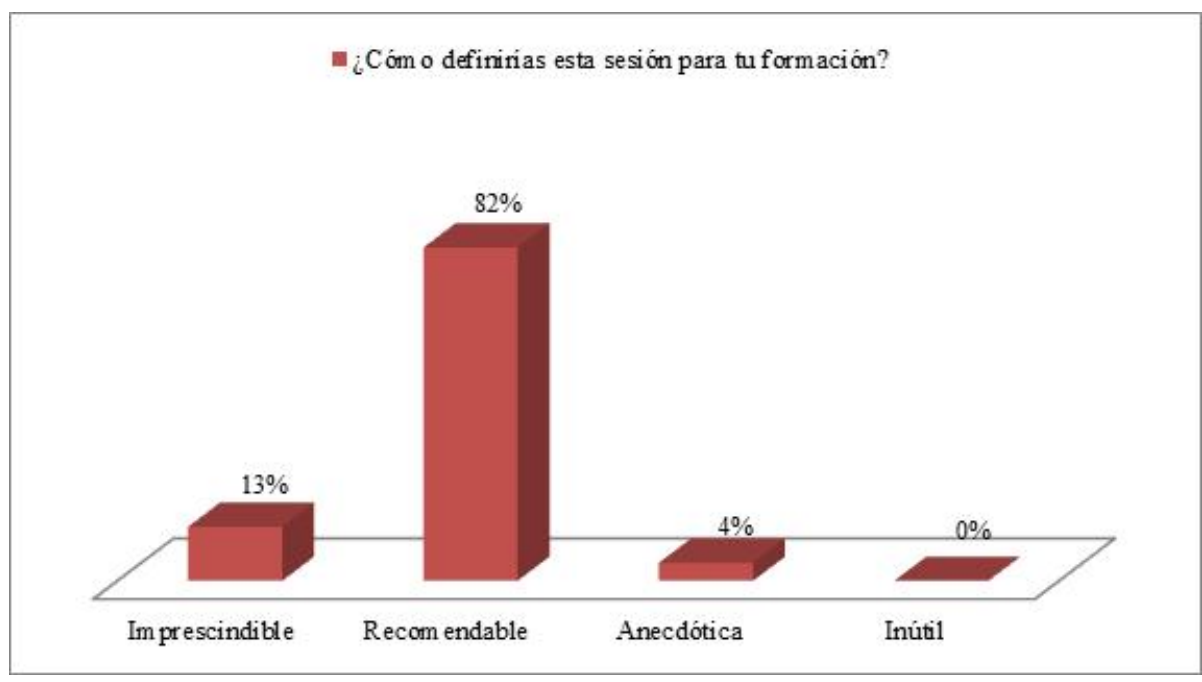

Fig 7. Valoración de los alumnos de la importancia de la sesión para su formación. 


\section{Conclusiones}

La primera conclusión que se confirma tras el análisis de las respuestas de los alumnos es la comprobación de que la formación en las aulas se centra generalmente en el aprendizaje de conocimientos teóricos y muchas veces abstractos. Esto conlleva un déficit a la hora de enseñar y trabajar los conocimientos más prácticos y sus aplicaciones. Se hace explícito en la encuesta de conocimientos previos y los conceptos mejor asimilados por lo estudiantes son los teóricos, como el frío y el calor, mientras que los relacionados con los prototipos de disipación presentan los niveles de entendimiento más bajos.

Se considera que para alcanzar un aprendizaje integral se debe dominar un concepto a todos los niveles, comprensión aplicación, relación, etc. Resulta llamativo que la mayoría de los alumnos consideren que conocen las diferencias entre los medios de disipación (65\%) pero no hayan visto nunca un prototipo de disipación (solo un 16\%). Estos resultados prueban la necesidad de profundizar en los conceptos desde todos los niveles de conocimiento para poder asegurar el proceso de aprendizaje completo.

Los datos analizados también demuestran que esta experiencia educativa fomenta el aprendizaje constructivo. A partir de las clases teóricas impartidas en el aula se completan y asimilan los conceptos en su totalidad con otro tipo de metodologías como las sesiones prácticas. El 100\% de los alumnos afirma conocer los conceptos de frío y calor antes de realizar la práctica, sin embargo, con la realización de la sesión un elevado porcentaje (96\%) considera que estos conocimientos se han afianzado y asimilado mejor. Estos datos confirman por tanto, la eficacia de la sesión para profundizar en los contenidos.

Por otra parte, se considera fundamental la realización de prácticas de este tipo que fomente en papel activo de los alumnos, donde ellos son los guías de su propio aprendizaje. Este tipo de herramientas educativas permiten desarrollar las competencia de aprender a aprender y de iniciativa y espíritu emprendedor, difíciles de integrar en clases teóricas y con mayor número de alumnos.

Los datos permiten también conocer de cerca la opinión de los alumnos sobre la sesión y sus propuestas de mejora. Un elevado porcentaje (82\%) considera que es una experiencia recomendable para su formación como arquitectos, un 13\% la considera imprescindible y tan solo un $4 \%$ la valora como anecdótica. Cabe destacar que ningún alumno ha considerado la sesión inútil, este dato confirma que la sesión realmente posee un valor educativo para los futuros arquitectos.

Por último, los alumnos han realizado diferentes propuestas de mejora en el último apartado de la encuesta, algunas de las más relevantes son: incluir el dibujo del esquema de los prototipos para facilitar su identificación, y poder profundizar en la tecnología manipulando más aplicaciones reales de las Peltier. Es también común la petición de más clases de este tipo y visitar otros prototipos a escala. Estas opiniones demuestran el interés y la motivación que genera la sesión en los alumnos y además, permite la mejora continua de la práctica ya que se pueden incluir las propuestas interesantes en la docencia de próximos cursos.

(cc) EY-NC-ND 2018, Universitat Politècnica de València

Congreso IN-RED (2018) 


\section{Agradecimientos}

Queremos agradecer el apoyo provisto por el proyecto BIA2013-46463- del Ministerio de Ciencia e Innovación del Gobierno de España y el proyecto 0011-1365-2016-000289 del Gobierno de Navarra.

También queremos agradecer a Jesús Maria Sánchez de Berotza por la ejecución de los equipos termoeléctricos.

\section{Referencias}

Amran, M. S., \& Rahman, S. (2017). The use of humour in mathematics teaching and its relationship with students' concentration and motivation. Advanced Science Letters, 23(2).

Ben Amara, F., \& Ayari, W. (2016). Team-based serious games and useful mathematics in engineering education. In International Symposium on Project Approaches in Engineering Education (Vol. 6).

Can, E. (2016). A different method proposal to improve of skills and success of the subtraction at primary schools in turkey. Turkish Online Journal of Educational Technology, 2016(NovemberSp).

De Menezes Maciel, A., Do Rêgo, R. G., \& Carlos, E. J. (2017). Pedagogic possibilities for using photographic images in didactic mathematics books | Possibilidades pedagógicas do uso da imagem fotográfica no livro didático de matemática. Bolema Mathematics Education Bulletin, 31(57).

Deniz, S., \& Simsek, I. (2015). The effect of 3D virtual learning environments on mathematic success: Second life sample. Turkish Online Journal of Educational Technology, 2015.

Felder, R. M., \& Brent, R. (2005). Understanding Student Differences. Journal for Engineering Education, 94(1), 57-72.

Felder, R., \& Silverman, L. (1988). Learning and teaching styles in engineering education. Engineering Education, 78(June), 674-681.

Granf, F., \& Marino, G. (2016). Building Environment and Interior Comfort in 20thCentury Architecture: Understanding Issues and Developing Conservation Strategies. Laboratory of Techniques and Preservation of Modern Architecture.

Ibañez-Puy, M., Bermejo-Busto, J., Martín-Gómez, C., Vidaurre-Arbizu, M., \& SacristánFernández, J. A. (2017). Thermoelectric cooling heating unit performance under real conditions. Applied Energy, 200, 303-314.

Ibañez-Puy, M., Martín-Gómez, C., Bermejo-Busto, J., Sacristán, J. A., \& Ibañez-Puy, E. (2018). Ventilated Active Thermoelectric Envelope (VATE): Analysis of its energy performance when integrated in a building. Energy and Buildings, 158, 1586-1592.

Ibáñez-Puy, M., Martín-Gómez, C., Vidaurre-Arbizu, M., \& Sacristán Fernandez, J. A. (2014). Theoretical Design of an Active Façade System with Peltier. Energy Procedia, 0, 0-4.

Ibañez-Puy, M., Sacristán Fernández, J. A., Martín-Gómez, C., \& Vidaurre-Arbizu, M. (2015). Development and Construction of a Thermoelectric Active Facade Module. Journal of Facade Design and Engineering, 3(1), 15-25. 
Martín-Gómez, C., Bermejo-Busto, J., \& Mambrilla-Herrero, N. (2015). Emergency lighting cabinet for fire safety learning. Case Studies in Fire Safety, 3, 17-24.

Martín-Gómez, C., Ramos, J. C., Rivas, A., Eguaras-Martínez, M., Mambrilla-Herrero, N., \& Torres, J. (2010). Prototype Thermoelectric Climate System For Its Use In Residential Buildings. In 29th International Conference on Thermoelectrics. Shangai, China. Retrieved from

Martín-Gómez, C., Zapata, O., Zuazua, A., Villanueva, S., \& Olaizola, P. (2014). Building services cabinets as teaching material in a degree in architecture. European Journal of Engineering Education, 39(2), 143-156.

Monedero Isorna, J. (2002). Enseñanza y práctica profesional de la arquitectura en Europa y EEUU-España. (Vol 2).

Vega-Moreno, D., Cufí Solé, X., José Rueda, M., \& Llinás, D. (2016). Integración de robótica educativa de bajo coste en el ámbito de la educación secundaria para fomentar el aprendizaje por proyectos Low cost educational robotics integration at high school scope to promote project- based learning. International Journal of Educational Research and Innovation, 6, 26-11. 